\title{
Prevalence and Associated Risk Factors of Pulmonary Tuberculosis in Diabetic Patients
}

\author{
P. V. R. Leelamohan ${ }^{\circledR 1}$, Mohan Kumar R ${ }^{\circledR 2}$ \\ ${ }^{1}$ Assistant Professor, Department of General Medicine, M. V. J. Medical College and Research Hospital, Bangalore, Karnataka, India, ${ }^{2}$ Associate Professor, Department of \\ General Medicine, Oxford Medical College, Bangalore, Karnataka, India.
}

\section{Abstract}

Background: One-third of India's population is infected with mycobacterium tuberculosis; there are 12 million active tuberculosis cases in India. One person dies of Tuberculosis every minute in India. No other disease has so much sociological, economic and health significance as Tuberculosis has. In 2005 there were an estimated 8.8 million new cases of Tuberculosis worldwide, with 1.9 million of those occurring in India. In India in 2000, there were an estimated 481,573,000 people over the age of 25 years. Among these, 4.3\% i.e. around 20,707,639 had diabetes, 19 and 939,064 developed. Subjects and Methods: The study conducted over a period of 2 yrs. Adult patients with diabetes mellitus with pulmonary Tuberculosis were included, while Diabetic patients with extrapulmonary Tuberculosis were excluded from the study. Results: The maximum incidence of pulmonary Tuberculosis was noted above the age of 30yrs were 13(26.0\%). The peak incidence was in the age group of $31-40$ and $41-50$ yrs. The mean age is 52.62. As Shown in [Figgure 1], the total numbers of males were $64 \%$ and females were $36 \%$. The male to female ratio was 2.11:1 As Shown in [Table 3], the distribution of patient according to their occupation noted were Skilled (18\%), Unskilled (38\%), Business (16\%), Service (2\%), Housewife (22\%), and Student (4\%). As shown in [Table 4], the socio-economic status among 50 patients was distributed into: Upper (2\%), Upper Middle (22\%), Upper lower (32\%), Lower (12\%), Lower middle (32\%), and Lower-middle (32\%). Conclusion: Early diagnosis and properly monitored treatment regimen is the only time tested answer to this problem. All diabetics at the initial diagnosis and every year must have an x-ray chest done. All diabetics with abnormal weight loss, unexplained cough, or sudden increase of insulin requirement should have sputum examination and chest $\mathrm{x}$-ray done.

Keywords: Ziehl-Neilsen Stain, Lowenstein -Jensen Medium, Bilateral Opacities, Mortality Rates

Corresponding Author: Mohan Kumar R, Associate Professor, Department of General Medicine, Oxford Medical College, Bangalore, Karnataka, India.

E-mail: dr.mohank@gmail.com

Received: 24 December 2019

Revised: 12 February 2020

Accepted: 5 March 2020

Published: 25 June 2020

\section{Introduction}

Tuberculosis is an ancient disease that continues to haunt us even as we step into the next millennium and it is one of the major public health problems in the developing countries of the world today. One-third of India's population is infected with mycobacterium tuberculosis. There are 12 million active tuberculosis cases in India. One person dies of Tuberculosis every minute in India. ${ }^{[1]}$ No other disease has so much sociological, economic and health significance as Tuberculosis has. In 2005 there were an estimated 8.8 million new cases of Tuberculosis worldwide, with 1.9 million of those occurring in India. In India in 2000, there were an estimated $481,573,000$ people over the age of 25 years. ${ }^{[2]}$ Among these, $4.3 \%$ i.e. around 20,707,639 had diabetes, 19 and 939,064 developed. Pulmonary Tuberculosis, of which 575,900 were smear-positive and hence infectious. ${ }^{[3]}$ Recent studies predict that in India, $18.4 \%$ (12.5\% to $29.9 \%)$ of people with pulmonary Tuberculosis have diabetes and that in the smear-positive group diabetes prevalence is $23.5 \%$ (12.1\% to 44\%). United Nations: World Population Prospects 2006. Various studies show that the incidence of Tuberculosis in diabetic patients varies from $1.6-8.4 \% .{ }^{[4]}$ Early diagnosis of the combination is rare. At the time of diagnosis, a large majority of cases have severe diabetes and far advanced pulmonary Tuberculosis. ${ }^{[5]}$ In a study from the regional institute of medical sciences, Imphal, the prevalence of pulmonary Tuberculosis in diabetics was found to be $27 \%$ by radiological diagnosis and $6 \%$ by sputum positivity. ${ }^{[6]}$ Rising prevalence of Tuberculosis in diabetics has been seen with age. Mortality rates in these patients are reported to be several times higher than in nondiabetic pulmonary tuberculosis patients. In a study from Congo, diabetes appeared to have an induction 
and aggravating effect on Tuberculosis. Irrespective of the triggering mechanisms, the fact remains that an epidemic of diabetes mellitus is sweeping the country. ${ }^{[7]}$ The recent prevalence data has propelled the estimates for India upwards32 million in 2000 and 80 million in 2030. This study has, therefore, been undertaken to determine the prevalence rate and associated risk factors of Tuberculosis in diabetes.

\section{Subjects and Methods}

All type 2 diabetic patients reporting to MVJMC and RH will form the subjects for study. The study conducted over a period of 2 yrs. Adult patients with diabetes mellitus with pulmonary Tuberculosis were included, while Diabetic patients with extrapulmonary Tuberculosis were excluded from the study. Diagnosis of diabetes was based on the National Diabetes Data Group and World Health Organization (WHO) which issued diagnostic criteria for diabetes mellitus based on the spectrum of fasting plasma glucose (FPG) and the response to an oral glucose load varying among normal individuals. Individuals with IFG or IGT are at substantial risk for developing type 2 diabetes mellitus ( $40 \%$ risk over the next 5 years) and cardiovascular disease. Oral glucose tolerance testing, although still a valid mechanism for diagnosing diabetes mellitus is not recommended as part of routine care. Diagnosis of Pulmonary Tuberculosis was made on the basis of sputum examination that was examined by direct smear using the Ziehl-Neilsen stain. The fluorescence method allows a large number of specimens to be examined rapidly. Sputum smear examination is usually positive in advanced disease but may be negative in less advanced disease, Sputum culture: Growth is visible on culture only after $3-8$ weeks (Lowenstein -Jensen medium). Rapid diagnosis is now possible within $2-6$ days using a radiometric culture system, the basic system. In any patient in whom the diagnosis is in doubt, repeated smear and culture examination should be carried out,

\section{Radiological appearances suggestive of Tuberculosis}

Following characteristics of a Chest radiograph favors the diagnosis of Tuberculosis:

1. Opacities mainly in the upper zone (s),

2. Patchy or nodular opacities,

3. Presence of cavity or cavities,

4. Presence of calcification,

5. Bilateral opacities especially if in upper zones,

6. Opacities that persists after several weeks (and thus are less likely due to acute pneumonia).

\section{Results}

Table 1 shows the age wise distribution of Patients. The maximum incidence of pulmonary Tuberculosis was noted above the age of 30 years were $13(26.0 \%)$. The peak incidence was in the age group of $31-40$ and $41-50$ years. The mean age is 52.62. As shown in Figure 1, the total numbers of males were $64 \%$ and females were $36 \%$. The male to female ratio was 2.11:1 As Shown in the Table 2 the distribution of patient according to their occupation noted were Skilled (18\%), Unskilled (38\%), Business (16\%), Service (2\%), Housewife (22\%), and Student (4\%). As shown in Table 3, the socioeconomic status among 50 patients was distributed into Upper (2\%), Upper Middle (22\%), Upper lower (32\%), Lower (12\%), Lower middle (32\%), and Lower-middle (32\%). As shown in the Table 4 , The predominant presenting symptoms noted were cough (76\%), expectoration ( $74 \%$ ), fever $(60 \%)$, in all the age groups. Significant percentage of patients had a loss of weight. As shown in Table $538 \%$ of the patients had a duration of diabetes between $6-10$ years and $22 \%$ of the patients had a duration of diabetes between $11-20$ years and in $36 \%$ of the patients, duration of the diabetes was less than 5 year. $4 \%$ of the patients had diabetes for more than 20 years. $40 \%$ of male patients in our study were smokers and $34 \%$ are alcoholics [Figure 2, Figure 3].

\begin{tabular}{|c|c|c|}
\hline Age in years & $\begin{array}{l}\text { Number of } \\
\text { patients }\end{array}$ & Percentage (\%) \\
\hline $31-40$ & 13 & 26.0 \\
\hline $41-50$ & 13 & 26.0 \\
\hline $51-60$ & 10 & 20.0 \\
\hline $61-70$ & 8 & 16.0 \\
\hline$>70$ & 6 & 12.0 \\
\hline Total & 50 & 100.0 \\
\hline
\end{tabular}

Mean \pm SD: $52.62 \pm 14.25$

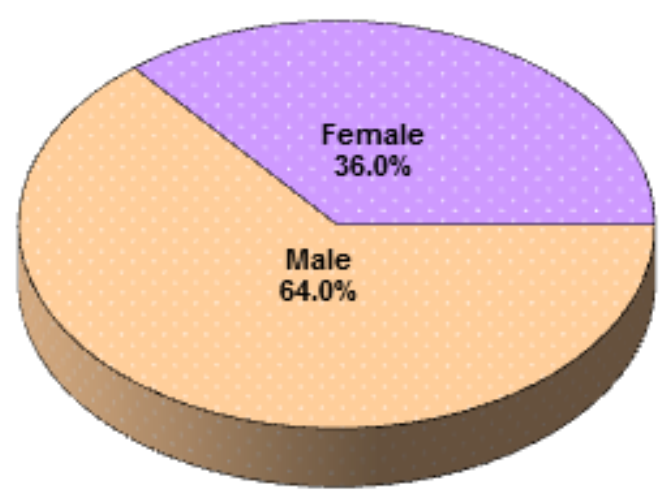

Gender 
Table 2: Distribution of occupation of patients studied

\begin{tabular}{|c|c|c|}
\hline Occupation & $\begin{array}{l}\text { Number of } \\
\text { patients }\end{array}$ & Percentage $(\%)$ \\
\hline Skilled & 9 & 18.0 \\
\hline Unskilled & 19 & 38.0 \\
\hline Business & 8 & 16.0 \\
\hline Service & 1 & 2.0 \\
\hline Housewife & 11 & 22.0 \\
\hline Student & 2 & 4.0 \\
\hline Total & 50 & 100.0 \\
\hline
\end{tabular}

Table 3: Distribution of socio-economic status of patients studied (SES)

\begin{tabular}{|c|c|c|}
\hline SES & $\begin{array}{l}\text { Number of } \\
\text { patients }\end{array}$ & Percentage $(\%)$ \\
\hline Upper & 1 & 2.0 \\
\hline Upper middle & 11 & 22.0 \\
\hline Upper lower & 16 & 32.0 \\
\hline Lower & 6 & 12.0 \\
\hline Lower middle & 16 & 32.0 \\
\hline Total & 50 & 100.0 \\
\hline
\end{tabular}

Table 4: Distribution Of Symptoms Of Patients Studied

\begin{tabular}{|c|c|c|}
\hline Symptoms & $\begin{array}{l}\text { Number of } \\
\text { patients }(n=50)\end{array}$ & Percentage $(\%)$ \\
\hline \multicolumn{3}{|l|}{ Fever } \\
\hline - No & 20 & 40.0 \\
\hline - Yes & 30 & 60.0 \\
\hline \multicolumn{3}{|l|}{ Cough } \\
\hline • No & 12 & 24.0 \\
\hline - Yes & 38 & 76.0 \\
\hline \multicolumn{3}{|c|}{ Expectoration } \\
\hline - No & 6 & 12.0 \\
\hline - Yes & 44 & 88.0 \\
\hline \multicolumn{3}{|c|}{ Breathlessness } \\
\hline - No & 19 & 38.0 \\
\hline - Yes & 31 & 62.0 \\
\hline \multicolumn{3}{|c|}{ Loss of weight } \\
\hline - No & 23 & 46.0 \\
\hline - Yes & 27 & 54.0 \\
\hline
\end{tabular}

\begin{tabular}{|c|c|c|}
\hline $\begin{array}{l}\text { Duration of dia- } \\
\text { betics }\end{array}$ & $\begin{array}{l}\text { Number of } \\
\text { patients }\end{array}$ & Percentage (\%) \\
\hline$<5$ & 18 & 36.0 \\
\hline $6-10$ & 19 & 38.0 \\
\hline $11-20$ & 11 & 22.0 \\
\hline$>20$ & 2 & 4.0 \\
\hline Total & 50 & 100.0 \\
\hline
\end{tabular}

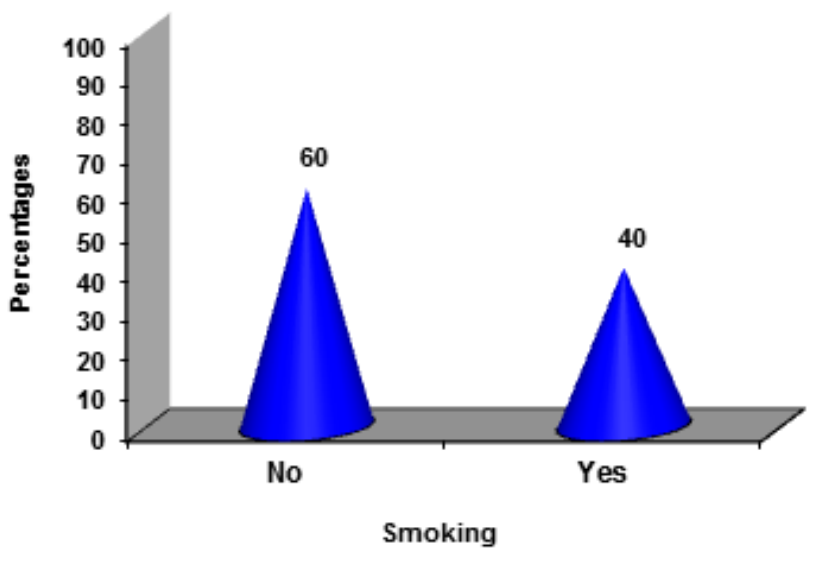

Figure 2: Distribution of Habit (Smoking) of Patients Studied

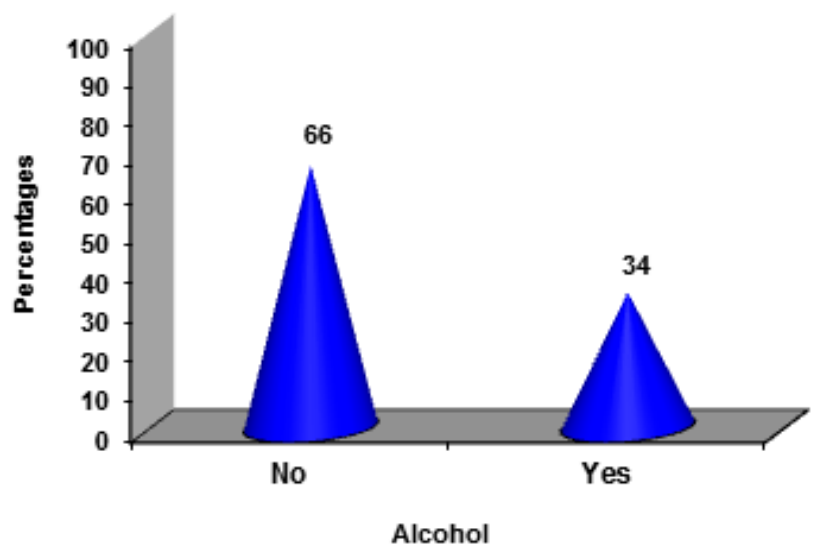

Figure 3: Distribution of Habit (Alcohol) of Patients Studied 


\section{Discussion}

This study was conducted on 50 patients who came with Tuberculosis and had Diabetes mellitus on investigations. Clinical and radiological evaluation of 50 cases of pulmonary Tuberculosis with diabetes mellitus was done. The high incidence of Tuberculosis in diabetic patients, reported by western and Indian workers, suggests a significant association between these two diseases. ${ }^{[8]}$ In the present study, the number of patients above the age of 30years was $13(26.0 \%)$ and the peak incidence was in the age groups of $31-40$ and $41-$ $50(26.0 \%) .50 \%$ of the total cases were in these age groups. The majority of cases of tuberculous diabetics belong to the age group of 45 yrs and above $(82.6 \%)$. ${ }^{[9]} 57.1 \%$ of the patients were above the age of $40 \mathrm{yrs} .{ }^{[10]}$ Half of the active new cases of Tuberculosis were observed in those who were at least $45 \mathrm{yrs}$ and relative incidence was highest in those who had passed 65 yrs. ${ }^{[11]}$ This study also showed a higher incidence of tuberculous diabetics in people over the age of 40 yrs. In this study the highest incidence was in the age group of $60-69 \mathrm{yrs}$. In our study the total number of males was $64 \%$ and females were $36 \%$. The male to female ratio was $2.11: 1$. Other studies have shown that, the prevalence as well as incidence of Tuberculosis is higher among males than among females, the ratio varying from 3:1 to5:1.In a study of 2434 cases of pulmonary Tuberculosis, it was reported that $62.9 \%$ were males and $37.1 \%$ were females. ${ }^{[12]}$ Among the 138 cases of diabetes with pulmonary Tuberculosis, $72.4 \%$ were males and $27.53 \%$ were females. ${ }^{[13]} \mathrm{A}$ similar ratio of male female. In the 179 cases he studied, $76 \%$ were males and $24 \%$ were females. ${ }^{[14]}$ In other study observed that male population out numbered the females. ${ }^{[15]} 78 \%$ of patients were males. ${ }^{[16]}$ All the above studies showed an increased incidence of the disease in males as compared to females and our study also noted the same. The high incidence of disease in males is possibly due to the fact that both Tuberculosis and diabetes are more common in males. Another reason could be that the number of male patients getting admitted to the hospital are more than females. In our study when socio-economic status of patients is considered the upper, lower, and lower-middle are $32 \%$ and $32 \%$ among the total patients. The same as well as other studies showed prevalence is common in low socioeconomic status. The role of diabetes mellitus in the higher prevalence of Tuberculosis.

When the occupation is taken in to picture in our study it is commonly found in unskilled patients $(38.0 \%)$ and next to them house wives $(22.0 \%)$. Other studies also show the same findings. ${ }^{[17]}$ The predominant symptoms noted in this study were cough (76\%) (No of patients -38) expectoration $(74 \%)$, (No of patients-36) fever (60\%) (No of patients- 30$)$ loss of weight (54\%) (No of patients-27). ${ }^{[18]}$ In a study done in Ethiopian diabetic patients with Tuberculosis, three most common symptoms of Tuberculosis were fever (80.5\%), sweating (80.4\%) and cough (70.5). ${ }^{[19]}$ In our study also a percentage of cough and fever was high associated with a history of weight loss in 27 patients among fifty patients. $20 \%$ of our patients had a past history of Tuberculosis. $10 \%$ of patients in our study had family history of Tuberculosis.20 out of 32 males $(40.0 \%)$ were smokers in our study. It has been shown that in patients of both sexes over the age of 30 with pulmonary Tuberculosis, there is a highly significant deficiency of nonsmokers and light smokers, and an excess of moderate and heavy smokers compared to controls of same age suffering from other diseases. ${ }^{20]}$

This study shows that $40.0 \%$ were smokers (males) and smoking may be a contributing factor in developing Tuberculosis in these patients. The study of the duration of diabetes in relation to the onset of Tuberculosis showed that, in most cases, Diabetes mellitus was diagnosed before the development of Tuberculosis. Prior to the onset of Tuberculosis, $38 \%$ patients had a duration of diabetes between 6-10years. 36\% patients had a duration of diabetes between less than 5 years, the average duration of diabetes in this study was $6.9 \mathrm{yrs}$ with SD 7.98+ 4.87. The interval between the detection of diabetes and the onset of pulmonary Tuberculosis reported that it varied from several months to $15 \mathrm{yrs}$, the mean interval being about 6 yrs, which is same to this study. ${ }^{[21]}$ In another study, diabetes was detected before Tuberculosis in $70 \%$ of cases. $32 \%$ of patients had diabetes for $1-5 \mathrm{yrs}, 32 \%$ had it for 6 $-10 \mathrm{yrs}$ and $20 \%$ more than $10 \mathrm{yrs} .{ }^{[22]}$ The duration of diabetes is significant because there is an increased opportunity for infections with increased duration of diabetes. The result of the present study is comparable to other studies. Some studies have reported that there is no correlation between the duration of diabetes and the development of pulmonary Tuberculosis.

\section{Conclusion}

Early diagnosis and properly monitored treatment regimen is the only time tested answer to this problem. All diabetics at the initial diagnosis and every year must have an $\mathrm{x}$-ray chest done. All diabetics with abnormal weight loss, unexplained cough, or sudden increase of insulin requirement should have sputum examination and chest $\mathrm{x}$-ray done.

\section{References}

1. Syal K, Srinivasan A, Banerjee D, Vdr, Rxr. Coronin-1 and interferon $r$ levels in PBMCs of type-2 diabetes patients: Molecular link between diabetes and Tuberculosis. Ind J Clin Biochem. 2015;30(3):323-328. Available from: https://dx.doi. org/10.1007/s12291-014-0431-7.

2. Diabetes and tuberculosis: the impact of the diabetes epidemic on tuberculosis incidence. BMC Public Health. 2007;7:234. Available from: https://dx.doi.org/10.1186/1471-2458-7-234. 
3. Smear positive pulmonary tuberculosis and its risk factors among tuberculosis suspect in South East Ethiopia; a hospital based cross-sectional study. BMC Res Notes. 2014;7:285. Available from: https://dx.doi.org/10.1186/1756-0500-7-285.

4. Jeon CY, Murray MB, Baker MA. Managing tuberculosis in patients with diabetes mellitus: why we care and what we know. Expert Rev Anti Infect Ther. 2012;10(8):863-868. Available from: https://dx.doi.org/10.1586/eri.12.75.

5. Riza AL, Pearson F, Ugarte-Gil C, Alisjahbana B, van de Vijver $\mathrm{S}$, Panduru NM, et al. Clinical management of concurrent diabetes and tuberculosis and the implications for patient services. Lancet Diab Endocrinol. 2014;2(9):740-753. Available from: https://dx.doi.org/10.1016/s2213-8587(14)70110-x.

6. Ezung T, Devi NT, Singh NT, Singh TB. Pulmonary tuberculosis and diabetes mellitus-a study. J Indian Med Assoc. 2002;100(6):378-379. Available from: http://whqlibdoc.who. int/publications/201/9789241502252 eng.pdf.Accessed.

7. Lin Y, Li L, Mi F, Du J, Dong Y, Li Z. Screening patients with diabetes mellitus for Tuberculosis in China. Trop Med Int Health. 2012;17(10):1302-1308.

8. Screening of tuberculosis patients for diabetes mellitus is feasible with the existing health system in India. J Family Med Prim Care. 2016;5(4):886-887. Available from: https: //dx.doi.org/10.4103/2249-4863.201159.

9. Group ITDS. Screening patients with Tuberculosis for diabetes mellitus in India. Trop Med Int Health. 2013;18(5):636-45. Available from: https://dx.doi.org/10.1111/tmi.12084.

10. Li L, Lin Y, Tan MF, Liang S, Guo B, C. Screening patients with Tuberculosis for diabetes mellitus in China. Trop Med Int Health. 2012;17(10):1294-1301. Available from: https: //dx.doi.org/10.1111/j.1365-3156.2012.03068.x.

11. Jeon CY, Harries AD, Baker MA, Hart JE, Kapur A, Lönnroth $\mathrm{K}$, et al. Bi-directional screening for tuberculosis and diabetes: a systematic review. Trop Med Int Health. 2010;15(11):13001314. Available from: https://dx.doi.org/10.1111/j.1365-3156. 2010.02632.x.

12. Lin $\mathrm{Y}$, Innes $\mathrm{A}, \mathrm{Xu} \mathrm{L}, \mathrm{Li} \mathrm{L}$, Chen $\mathrm{J}$, Hou $\mathrm{J}$, et al. Screening of patients with diabetes mellitus for tuberculosis in community health settings in China. Trop Med Int Health. 2015;20(8):1073-80. Available from: https://dx.doi.org/10. $1111 /$ tmi.12519.

13. Jeon CY, Murray MB. Diabetes Mellitus Increases the Risk of Active Tuberculosis: A Systematic Review of 13 Observational Studies. PLoS Med. 2008;5(7):e152-e152. Available from: https://dx.doi.org/10.1371/journal.pmed.0050152.

14. Baker MA, Harries AD, Jeon CY, Hart JE, Kapur A, Lönnroth $\mathrm{K}$, et al. The impact of diabetes on tuberculosis treatment outcomes: A systematic review. BMC Med. 2011;9(1):81-85.
Available from: https://dx.doi.org/10.1186/1741-7015-9-81.

15. Achanta S, Tekumalla RR, Jaju J, Purad C, Chepuri R, Samyukta R. Screening tuberculosis patients for diabetes in a tribal area in South India. Public Health Action. 2013;3(1):4347. Available from: https://dx.doi.org/10.5588/pha.13.0033.

16. Alavi SM, Khoshkhoy MM. Pulmonary tuberculosis and diabetes mellitus: Co-existence of both diseases in patients admitted in a teaching hospital in South West of Iran. Caspian J Intern Med. 2012;3(2):421-424.

17. Alisjahbana B, Crevel RV, Sahiratmadja E, Heijer MD, Maya A, Istriana E. Diabetes mellitus is strongly associated with Tuberculosis in Indonesia. Int $\mathrm{J}$ Tuber Lung Dis. 2006;10(6):696-700.

18. Baghaei P, Tabarsi P, Marjani M, Moniri A, Masjedi MR. Screening for diabetes mellitus in tuberculosis patients in a referral center in Iran. Inf Dis. 2015;47(7):472-476. Available from: https://dx.doi.org/10.3109/23744235.2015.1018317.

19. Balakrishnan S, Vijayan S, Nair S, Subramoniapillai J, Mrithyunjayan S, Wilson N, et al. High Diabetes Prevalence among Tuberculosis Cases in Kerala, India. PLoS One. 2012;7(10):e46502-e46502. Available from: https://dx.doi. org/10.1371/journal.pone.0046502.

20. Dave P, Shah A, Chauhan M, Kumar AMV, Harries AD, Malhotra S, et al. Screening patients with tuberculosis for diabetes mellitus in Gujarat, India. Public Health Action. 2013;3(1):29-33. Available from: https://dx.doi.org/10.5588/ pha.13.0027.

21. Jawad F, Shera AS, Memon R, Ansari G. Glucose intolerance in pulmonary Tuberculosis. J Park Med Assoc. 1995;45:237238.

22. Lin YH, Chen CP, Chen PY, Huang JC, Ho C, Weng HH. Screening for pulmonary Tuberculosis in type 2 diabetes elderly: a cross-sectional study in a community hospital. BMC Public Health. 2015;15:3-6.

Copyright: (C) the author(s), 2020. It is an open-access article distributed under the terms of the Creative Commons Attribution License (CC BY 4.0), which permits authors to retain ownership of the copyright for their content, and allow anyone to download, reuse, reprint, modify, distribute and/or copy the content as long as the original authors and source are cited.

How to cite this article: Leelamohan PVR, Kumar R M. Prevalence and Associated Risk Factors of Pulmonary Tuberculosis in Diabetic Patients. Acad. J Med. 2020;3(1):47-51.

DOI: dx.doi.org/10.47008/ajm.2020.3.1.10

Source of Support: Nil, Conflict of Interest: None declared. 